Images Re-vues

re-VUES

Histoire, anthropologie et théorie de l'art

$8 \mid 2011$

Figurer les invisibles

\title{
Images défuntes (Z32, Avi Mograbi, 2008)
}

\section{Emmanuelle André}

\section{(2) OpenEdition}

\section{Journals}

Édition électronique

URL : http://journals.openedition.org/imagesrevues/486

DOI : 10.4000/imagesrevues.486

ISSN : 1778-3801

\section{Éditeur :}

Centre d'Histoire et Théorie des Arts, Groupe d'Anthropologie Historique de l'Occident Médiéval, Laboratoire d'Anthropologie Sociale, UMR 8210 Anthropologie et Histoire des Mondes Antiques

\section{Référence électronique}

Emmanuelle André, «Images défuntes (Z32, Avi Mograbi, 2008)», Images Re-vues [En ligne], 8| 2011, mis en ligne le 20 avril 2011, consulté le 02 mars 2021. URL : http://journals.openedition.org/ imagesrevues/486 ; DOI : https://doi.org/10.4000/imagesrevues.486

\section{(9) $(1) \Theta$}

Images Re-vues est mise à disposition selon les termes de la Licence Creative Commons Attribution Pas d'Utilisation Commerciale 4.0 International. 


\title{
IMAGES DEFUnTES (Z92, Avi MogRABI, 2008)
}

\author{
Emmanuelle André
}

\begin{abstract}
Z 32 est le nom de code d'un soldat israélien qui, au cours d'une opération de représailles dans les territoires occupés, assassine un policier palestinien. Deux ans plus tard, il rencontre Avi Mograbi et accepte de raconter son histoire devant la caméra, à condition que son identité reste cachée. Afin de donner au mal la forme humaine d'une transformation, le cinéaste met au point un masque numérique évolutif, qui renoue avec les origines de la figure à travers plusieurs pratiques et traditions anciennes, masque mortuaire et portrait votif. Au problème politique soulevé par Mograbi - comment faire face aux crimes de guerre? - le film instaure alors une dialectique entre le vu et le nonvu qui oppose à la mémoire brûlante du soldat la mémoire visuelle de l'histoire de l'art que l’innovation technologique fait affleurer à la surface, opacifiée, du visage.
\end{abstract}

«En même temps, le visage est ce qui nous interdit de tuer.» Emmanuel Levinas ${ }^{1}$

Rendre sensible un problème politique qui, pour s'inscrire dans une situation historique et géographique précise, n'en relève pas moins d'un questionnement éthique à visée plus générale: la reconquête d'une humanité perdue dans l'exécution d'un acte meurtrier. Tel est l'enjeu du film Z32, réalisé en 2008 par le cinéaste Avi Mograbi. Le titre renvoie au nom de code d'un ancien soldat israélien donné par l'association Shovrim Shtika («Briser le silence»), une organisation qui recueille les témoignages d'anciens militaires ayant servi dans les territoires occupés. Celui-ci a vingt ans quand il est enrôlé par son unité d'élite dans une opération de représailles. Cette nuit-là, le jeune homme, préparé au combat depuis des mois, assassine froidement un policier palestinien innocent. Deux ans plus tard, il accepte de raconter son histoire devant la caméra, à condition cependant que son identité reste cachée, par peur d'être victime d'un acte de vengeance ou d'être saisi par la justice internationale.

Pour cacher ce visage, plusieurs solutions s'offraient au cinéaste : il aurait pu, comme c'est l'usage dans les médias, marquer d'un bandeau noir les yeux du soldat ou encore le tenir hors du champ représenté. Il dit lui-même qu'il a pensé lui faire porter un vrai masque, mais que cela ne fonctionnait pas car il fallait que soit maintenue l'expression du visage. D'où l'idée d'utiliser l'infographie pour dissimuler les traits tout en maintenant découverts les yeux et la bouche, «de telle sorte que l'identité du garçon soit cachée mais ses sentiments révélés au regard $»^{2}$, explique Avi Mussel, le concepteur graphique. Afin de donner au mal la forme humaine d'une transformation, le cinéaste met au point un masque numérique évolutif, «qui cache l'identité et en même temps permet au spectateur [...] de voir qu'il s'agit d'un être humain $»^{3}$. Ce masque prend trois aspects successifs au cours du film. Le premier est un «ovale de fumée $»^{4}$, qui dans certains plans envahit la totalité du champ: l'impression provoquée est celle d'une déformation inquiétante et monstrueuse (fig.1). Le deuxième simule le papier mâché et marque clairement la limite du visage (fig.2). Le troisième a été

\footnotetext{
${ }^{1}$ Emmanuel Levinas, Éthique et Infini, Fayard, Radio-France, 1982, p. 80.

2 Avi Mussel, « Dissimuler pour révéler l'effet de regard », Trafic 72, hiver 2009, p. 56-63.

${ }^{3}$ Avi Mograbi, entretien paru dans les Cahiers du Cinéma 639, novembre 2008, p. VI et VII.

${ }^{4}$ Selon l'expression d'Avi Mograbi, Ibidem.
} 
conçu sur le modèle de la greffe photographique et produit l'étrange effet d'un vrai visage, n'était le passage de la main vers la bouche, qui confirme la présence d'un voile numérique (fig.3).

Fig.1.

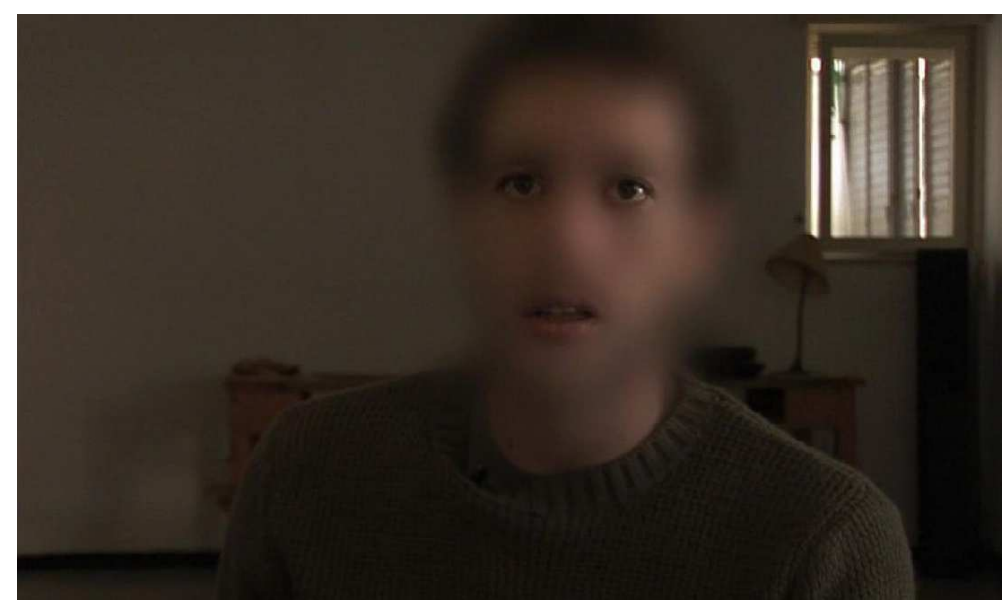

Avi Mograbi, Z32, 2008 - Photogramme du film (dvd)

Fig.2.

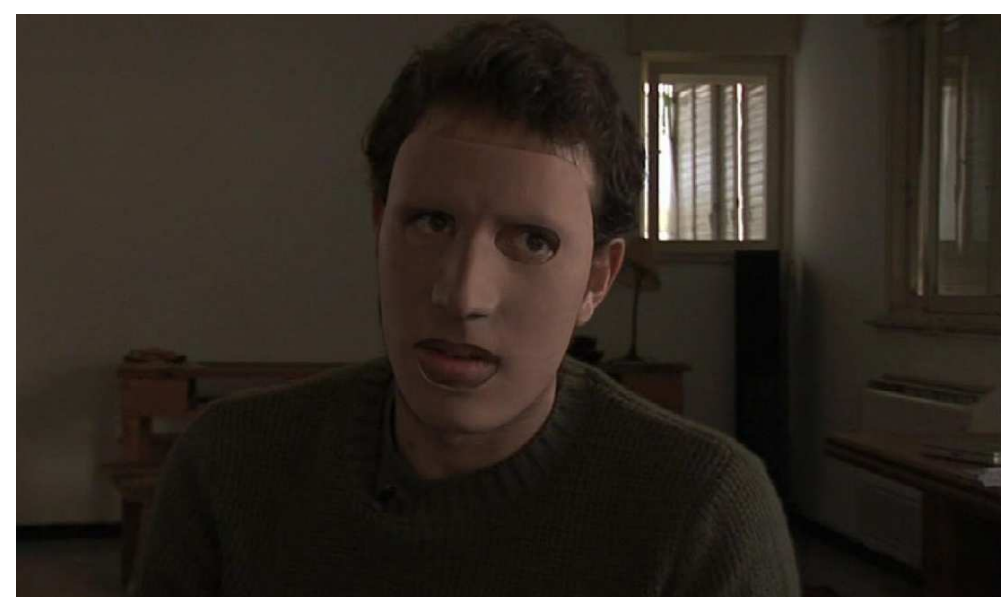

Avi Mograbi, Z32, 2008 - Photogramme du film (dvd)

Fig.3.

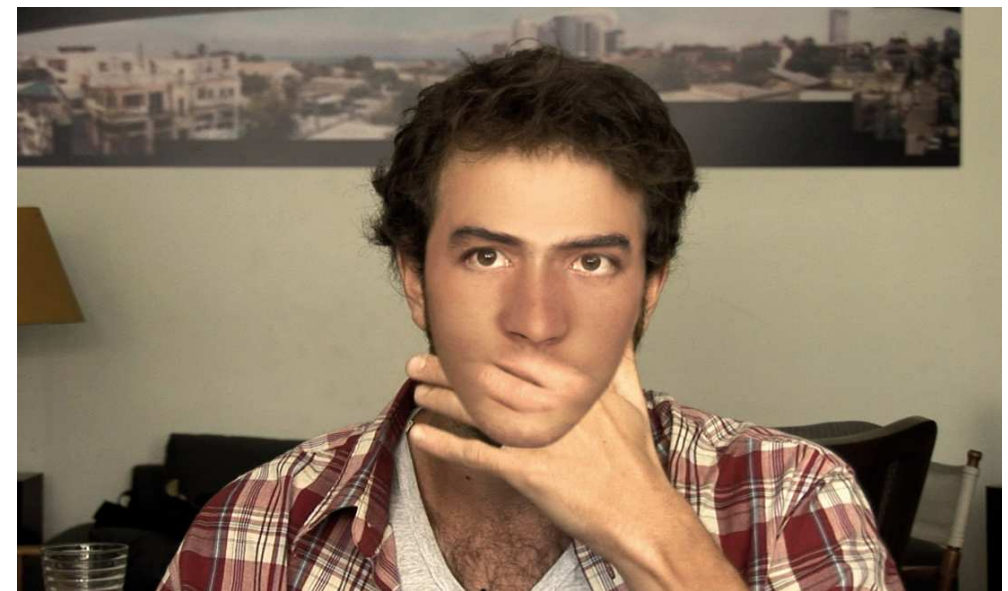

Avi Mograbi, Z32, 2008 - Photogramme du film (dvd) 
Un trajet se dessine alors, dans le temps du modelage numérique mis au service du déroulement cinématographique, qui ravive le travail de la figure: non plus figurer pour donner forme à une matière, mais défigurer pour redonner un visage d'homme. Autrement dit, il s'agit de laisser advenir sur le visage la part d'humanité que le récit lui retranche et d'instaurer une dialectique entre le vu et le non-vu: sans cesse montrer que, sur ce visage, quelque chose résiste à la vue - toujours, l'événement restera invisible à la représentation et configurer numériquement cette résistance. La contrainte imposée aux techniciens est scrupuleusement respectée. Au terme de neuf mois de travail, les effets spéciaux sont au point, qui réussissent à cacher l'identité tout en exprimant une vérité du sujet, dont les yeux et la bouche sont les signes saillants. Ainsi, au problème politique soulevé par Mograbi comment faire face aux crimes de guerre? - et à la question anthropologique qui lui est liée - comment envisager l'homme pour qu'il reconquière une humanité perdue ? - le film propose de dégager une forme de portrait qui brouille non pas seulement le rapport du visage à la semblance, mais convoque les catégories du visible et de l'invisible, en conjuguant plusieurs pratiques et traditions anciennes. À la mémoire du soldat, trop brûlante pour qu'il s'en débarrasse, le film oppose la mémoire visuelle de l'histoire de l'art que l'innovation technologique fait affleurer à la surface, opacifiée, du visage.

Peut-on faire d'un meurtrier le personnage principal d'un film, sans jamais lui donner l'occasion de justifier son geste, totalement condamnable? À ce point, le projet politique est rapporté à une question éthique, transformée en un problème de mise en scène : comment filmer le récit d'un acte intolérable et quelle place visible réserver à un tel narrateur? Devant l'actualité du conflit et l'urgence de sa résolution, le film réactive le dilemme de la ressemblance à l'œuvre dans la figuration du portrait, à partir d'un paradoxe : si le portrait, tel que glorifié par la tradition humaniste de la Renaissance, raccorde l'humanité d'une expressivité à l'identité d'un sujet, comment faire le portrait d'une perte, d'une perte d'humanité et en même temps de sa reconquête?

Comme le titre du film l'indique, l'identité du soldat s'est réduite à celle d'un matricule. Z32 ne nomme pas, c'est même le contraire d'un nom propre, qui indique l'inscription d'un élément dans une chaine numérique et évoque la mécanisation de la guerre, décrite en détails par le jeune homme, à travers les gestes d'attente, d'apprentissage et de préparation au combat. Si, comme le remarque Jean-Luc Nancy, «l'état civil du portrait, c'est son état figural $»^{5}$, alors ce titre dit la figurabilité d'une non-coïncidence entre l'attachement du soldat à son identité et à sa nation, à son armée. Or, la figurabilité de Z32, soit la possibilité, politique, d'une ressemblance, procède d'une démarche réflexive que le film déploie par une mise en scène des conditions même de la mise en scène. La description de la personne est ici totalement imbriquée dans la fabrique du portrait, dans toute sa complexité, technique, figurative et morale. D'abord sous une forme d'apparence très simple : « Comment héberger un assassin dans mon film? »- ne cesse de répéter Mograbi, qui joue son propre rôle. Mais pas seulement. Pour dire le geste manuel à l'origine de la fabrique, le film renvoie aux origines de la figure, avec laquelle le numérique renoue.

\section{Figura, figurae}

Erich Auerbach l'a rappelé une fois pour toutes ${ }^{6}$ : la figura est d'abord un objet moulé, une forme plastique, ainsi que le résultat du travail de la main sur la matière, son moulage et son modelage, dont le film restitue à la fois le geste et le temps, à travers la succession des trois visages, et les transformations de chacun des trois masques, très voyantes ou plus

\footnotetext{
${ }^{5}$ Jean-Luc Nancy, Le Regard du Portrait, Galilée, coll. « Incises », 2000, p. 23.

${ }^{6}$ Erich Auerbach, Figura (1944), (tr. fr.), Paris, Belin, coll. « L'extrême contemporain », 1993.
} 
subtiles. D'emblée, le film débute par l'exposition du jeune couple, le soldat et sa compagne, le visage totalement embué, comme dans un reportage télévisé. Le cinéaste apparaît ensuite dans son appartement, assis face à la caméra en plan rapproché. Une cagoule noire sur la tête, il lit le récit de son personnage, et explique bientôt pourquoi il est impossible de procéder de la sorte. Alors, avec une paire de ciseaux, il troue ce masque improvisé à la place des yeux, de la bouche et du nez (pour respirer) - (fig.4). Conséquence directe de cette curieuse manière de faire, la scène suivante montre en très gros plan, cernés d'un halo flou, l'œil puis la bouche du soldat (premier masque), alors qu'il raconte son histoire (figs.5 et 6) : son enrôlement en 1999 dans une unité d'élite après un an de service civil, l'intégration de la patrouille Yaël, les vingt mois d'entraînement.

Fig.4.

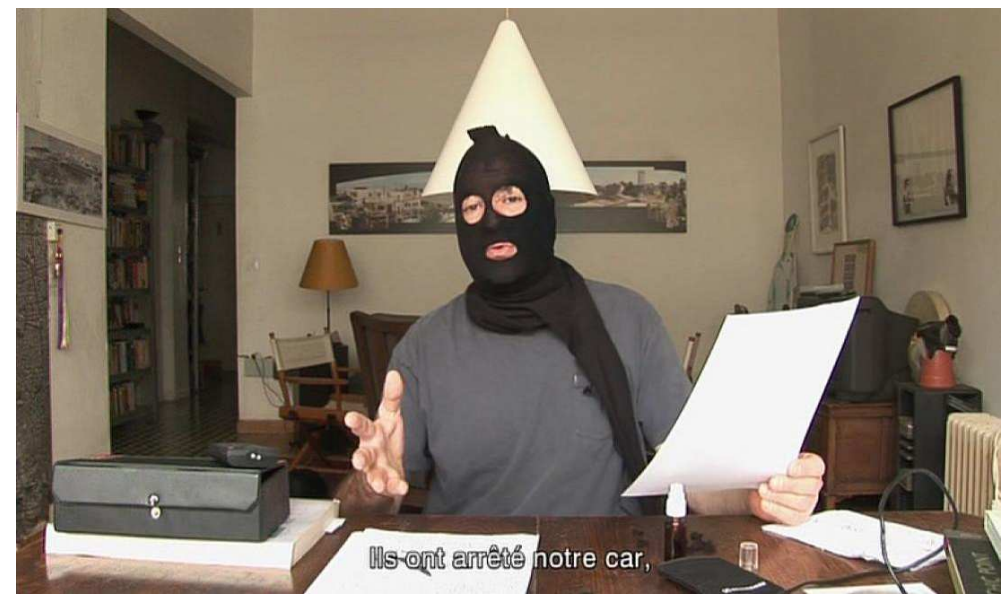

Fig.5.

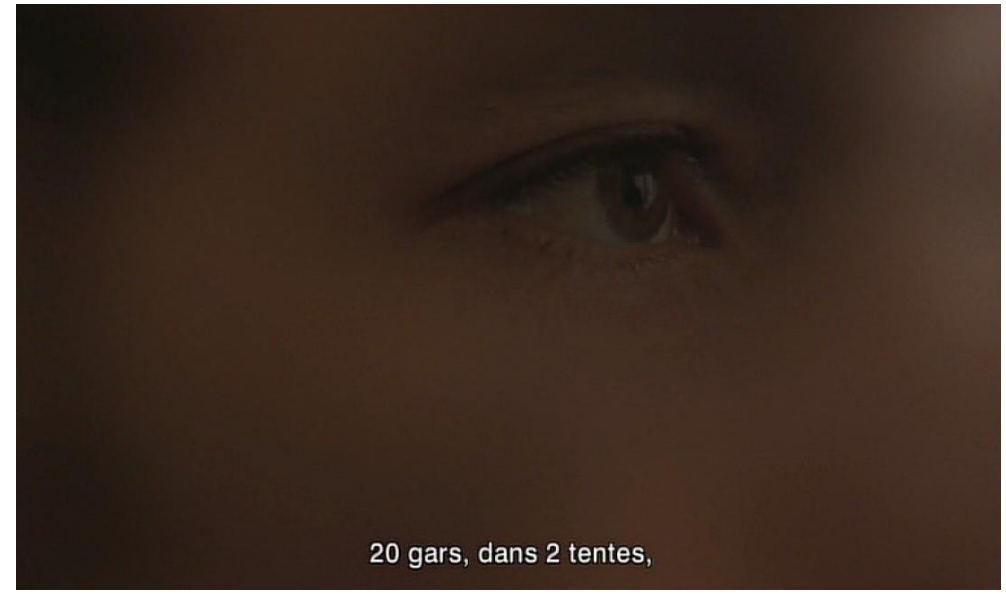

Avi Mograbi, Z32, 2008 - Photogrammes du film (dvd) 
Fig.6.

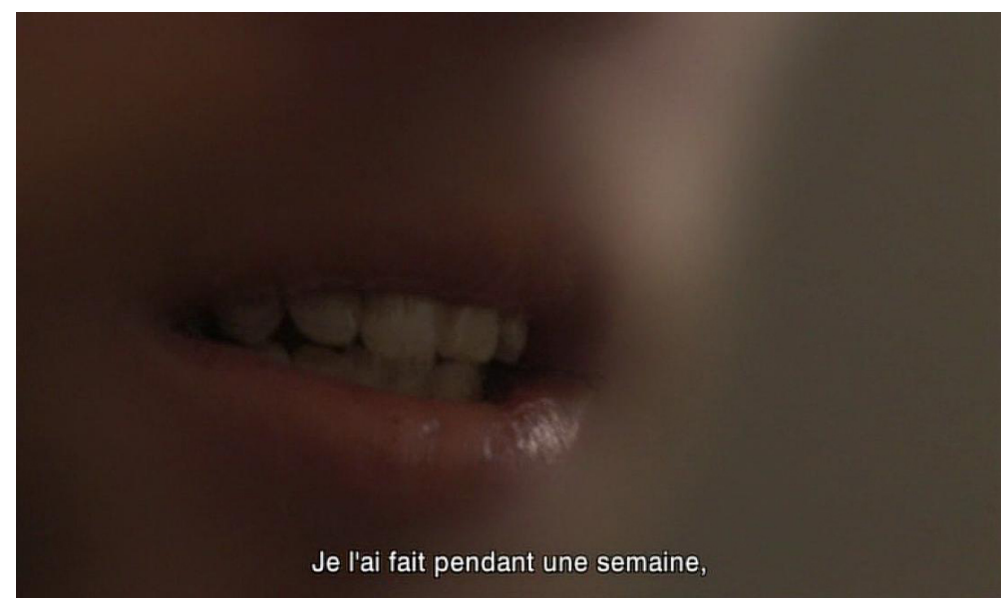

Avi Mograbi, Z32, 2008 - Photogramme du film (dvd)

Le récit se poursuit, mais le cadrage, de nouveau, change. Nous retrouvons la jeune fille, puis le jeune homme dans une petite pièce aux murs nus; ils sont cadrés en plan rapproché, le regard dirigé vers les hors-champ, où l'on devine un interlocuteur qu'on entend à peine une ou deux fois, mais qu'on ne voit pas. Désormais, le flou produit une sorte de guirlande qui entoure leur tête et laisse place à des trous singulièrement creusés, comparativement au reste du visage. Le ton employé est le même, d'une monotonie qui tranche avec les événements décrits et la forme de suspens qu'ils instillent, mais un brusque zoom avant fait événement, qui modifie une fois de plus la figure : le flou a pris possession du champ, il aplatit l'image et aplanit la face. L'œil et la bouche ne sont plus autrement cernés que par ce brouillard qui mange tout (fig.7). La netteté du discours en devient monstrueuse. Ainsi, le flou modèle le visage, il agit sur ses bordures et sur son relief, de même qu'il ne cesse de déplacer les rapports entre la forme de l'ovale et le fond du champ, avec lequel parfois il se confond. Bientôt, un deuxième masque surgit, d'un aspect totalement opposé, d'apparence rigide et épaisse, et d'une clarté qui gomme toutes les aspérités - sorte de moule destiné à la saisie d'une empreinte. Enfin, dans la dernière partie du film, une troisième transformation de la face est opérée. Désormais, une peau numérique restaure la ressemblance d'un très jeune homme, à peine sorti de l'enfance. C'est maintenant que le pire épisode de l'événement est rapporté : la mise à mort du policier.

Fig.7.

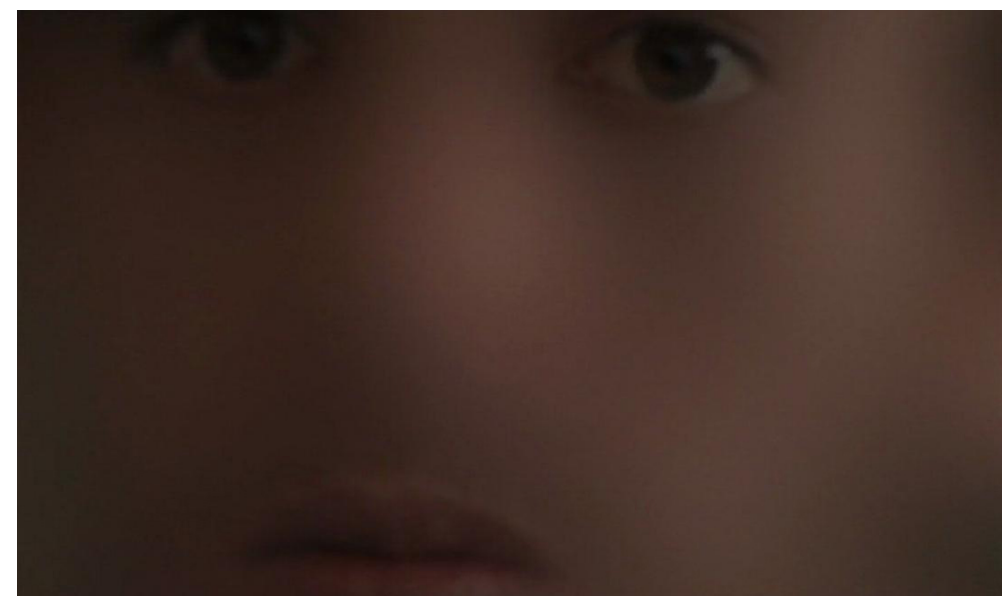

Avi Mograbi, Z32, 2008 - Photogramme du film (dvd)

On le voit, le numérique est paradoxalement utilisé pour ses qualités de matérialité plastique. Précisément, l'image virtuelle évoque la plasticité de la cire, qui suppose labilité et 
modelage du matériau. On sait que la cire a trouvé un terrain d'élection avec le visage, comme en témoigne l'enquête philologique sur le mot menée par Julius von Schlosser? Entre la fin de l'Antiquité et le bas Moyen Âge, le portrait réaliste en cire a laissé des traces linguistiques dans les langues romanes. L’italien cera serait dérivé du français chair «cara» (et de l'ancien français chière, chire), également employé en espagnol et en portugais pour dire la mine et le visage; on suppose qu'il dérive de kara, le mot grec ancien pour désigner la « tête », utilisé par Homère et les poètes tragiques et disparu ensuite dans les langues vivantes. Il est fort probable que cette rencontre sémantique s'explique par l'usage de la cire pendant l'Antiquité où elle est mise au service de l'expressivité du visage dont, mieux qu'aucun autre matériau elle assure la conservation. C'est bien le numérique dans le film qui permet de «tirer » le portrait, à savoir une vérité de l'homme, que fait resurgir le souvenir de la cire, à l'origine de ce caractère - expressif - de la personne.

La figure elle-même est le visage, la face, mais avant que ce sens-là ne se fixe, elle a désigné la feinte que le masque personnifie, figura - de la même famille que fingere, feindre renvoyant aussi à la fiction. Jean-Marie Pontévia rappelle ce pouvoir figuratif du visage («faire bonne ou mauvaise mine ») qui explique qu'il est toujours déjà dessiné, comme l'exprime aussi le verbe portraire ; «cela signifie - précise Pontevia - qu'il est de l'ordre du dessin, de la projection d'une figure : le visage peut se faire surface à regarder et à déchiffrer ; le masque, le fard témoignent de ce trait du visage $»^{8}$. Voilà justement ce qui fait lien : parce qu'il révèle tout autant qu'il dissimule, le masque de Z32 préfigure le portrait, il ne cesse d'en parfaire l'esquisse car le trait est en échange constant avec le matériau. "Qu'est-ce qui fait un portrait? - ajoute Pontevia - C'est une certaine connivence du trait et des traits, l'instantané qui fixe la rencontre - mieux : l'échange - qui voit le trait (du dessin) devenir vivant et les traits (d'un visage) devenir graphiques : le visage est perçu "graphiquement" et il ne peut l'être qu'à la faveur d'un dessin $»^{9}$. N'est-ce pas là ce qui se joue dans le film lorsqu'un geste de la main vient soudainement redessiner les contours du dernier visage, laissant apparaître qu'il s'agit d'un faux, c'est-à-dire d'un masque (fig.8). Tout à coup, le contour de la figure surgit qui révèle le subterfuge par un effet de transparence que la main souligne - et que rappelle la fumée de cigarette qui s'échappe de la vraie bouche. Encore une fois, c'est bien la fabrique du portrait qui importe, dans le geste projectif du tracé, que la présence de la main tout près du visage remémore. Parce qu'il figure une origine de la figure, ce motif - le masque devant/derrière le portrait - préexiste au film, dans ce dessin de Magritte, par exemple, justement dénommé Tête-masque (fig.9). Comme le remarque Michel Servière :

L'ovale du visage [...] est si bien marqué jusqu'à seulement, qui l'irrégularise, la trace de l'implantation des cheveux absents de cette femme, les doigts et la cigarette sont si nettement coupés que, sans forcer pourtant, sans démenti linguistique dûment noté, ceci non plus n'est pas une tête. Mais un doux masque. Le masque: sur-face. Ceci est un Magritte encore, avouant sa platitude de feuille de dessin. Un portrait est toujours repassé sur papier ou toile tendue ${ }^{10}$.

\footnotetext{
7 Julius von Schlosser, Histoire du Portrait en Cire, Paris, Macula, coll. "La littérature artistique », [1911], 1997, p. 39-41.

${ }^{8}$ Jean-Marie Pontevia, "Ogni dipintore dipinge sè". Écrits sur l'Art et Pensées Détachées, vol. III, Bordeaux, William Blake \& Co., 1986, p. 11. Pour une approche théorique de l'histoire du portrait, voir Edouard Pommier, Théories du Portrait. De la Rennaissance aux Lumières, Paris, Gallimard, Bibliothèque illustrée des Histoires, 1998. En adoptant une démarche pluridisciplinaire, l'ouvrage de Françoise Frontisi-Ducroux (Du Masque au Visage. Aspects de l'identité en Grèce ancienne, Paris, Flammarion, coll. «Idées et recherches », 1995), qui s’inscrit dans la continuité des travaux de Jean-Pierre Vernant, insiste sur l'idée que le masque révèle l'identité au lieu de la cacher.

${ }_{9}$ Ibid., p. 13.

${ }^{10}$ Michel Servière, «L’imaginaire signé. Référence et sujet dans le portrait », in Eric Van Casteele, Jean-Louis Déotte, Michel Servière, Portrait, Autoportrait, Paris, Éditions Osiris, 1987, p. 104.
} 
Fig.8.

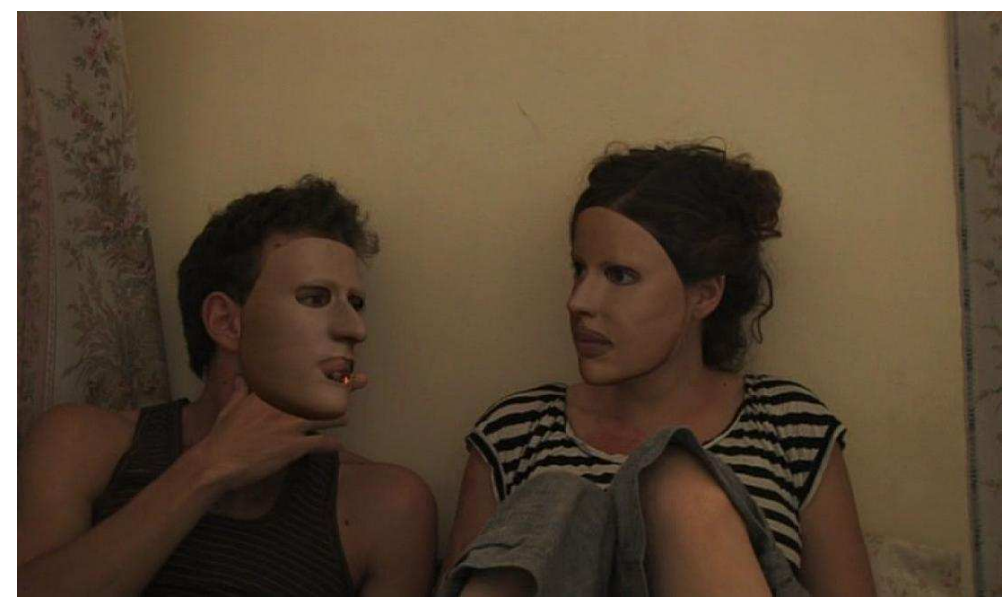

Avi Mograbi, Z32, 2008 - Photogramme du film (dvd)

Fig.9.

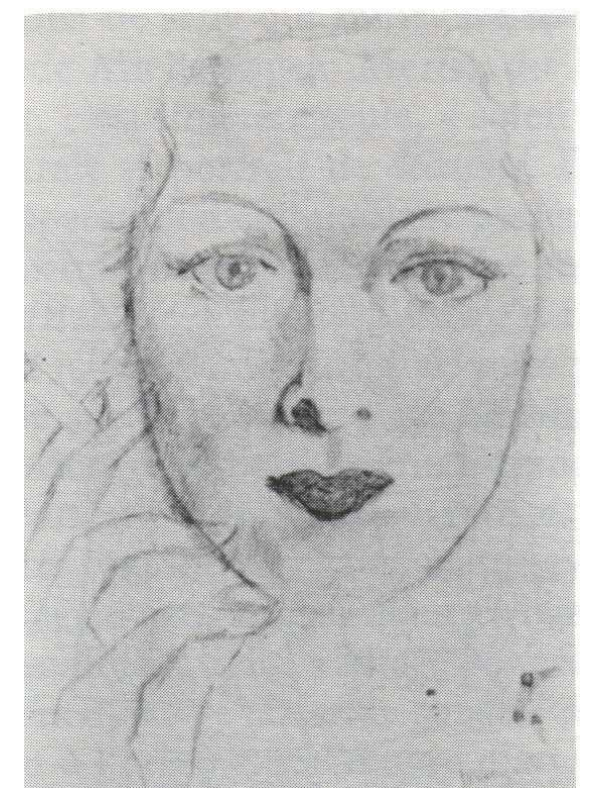

René Magritte, Tête-masque, dessin non daté

Dans le film, le numérique a la souplesse de faire déborder la platitude du visage sur la totalité du champ (au début, quand le visage est encore flou) et un peu après de produire un effet d'illusion si fort qu'il faut un mouvement de la main pour que le visage reprenne l'apparence de son origine - virtuelle : une feuille de dessin sur laquelle un dessin est tracé.

With my Tongue in my Cheek (Marcel Duchamp, 1959) reconduit le lien entre dessin et moulage. Un visage dessiné de profil est recouvert d'un moule en plâtre légèrement décalé par rapport à lui et n'occupant pas toute sa surface. Le contraste est frappant entre la finesse du trait qui se détache à peine de la surface plane du papier et la plénitude de la matière qui évoque celle de la chair (fig.10). En un même motif sont montrées la platitude de la figure et la mise en forme du visage, dont l'assemblage produit l'idée d'une transformation en cours et l'effet conséquent d'une puissante expressivité - mise en ouvre dans Z32 par le modelage temporel de la céroplastie virtuelle. Ces deux oeuvres activent une tension entre deux états superposés du visage; elles disent le processus de fabrication et la place de l'expressivité dans cette fabrique - dans la main chez Magritte, dans l'œeil et le regard perçant chez Duchamp - que le film tout à la fois rejoue et déplie linéairement, en le déplaçant : «Cachez- 
lui le visage pour pouvoir lui parler - chante le cinéaste - Laissez un trou pour le nez et les yeux pour deviner son sourire. Il dit qu'il faut laisser derrière lui son passé [...] Il a des raisons d'avoir peur [...] Il n'est après tout qu'un de ces figurants [...] Cachez-lui son visage pour pouvoir continuer à l'imaginer ». Le manque à combler entre deux aspects du visage - qu'évoquent dans un cas la superposition du masque, dans l'autre la couverture partielle de plâtre - ont chez Mograbi une signification politique : afin que le soldat ne soit pas qu'un pion (un figurant) pris dans l'engrenage de la guerre, mais un homme doué de raison qui veut comprendre son geste, une fiction du visage reste à réinventer.

Fig.10.

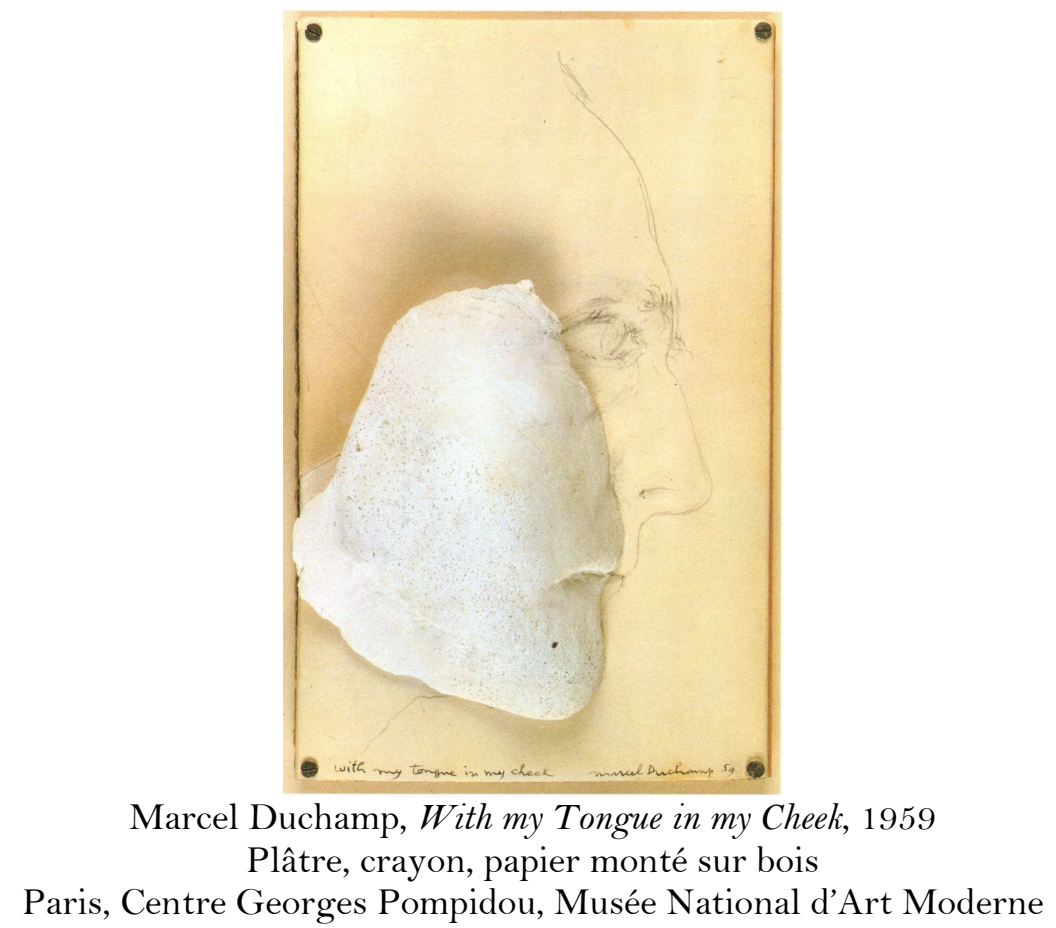

\section{Au vif de la matière}

Le masque (r)appelle le dessin et l'origine du portrait, que l'usage du numérique rend imaginable. Or, cette manière de remonter au geste de modelage renvoie à une pratique de l'histoire de l'art, à laquelle le film est lié : le portrait sur le vif. De quoi s'agit-il ? D'exécuter l'œuvre d'après un modèle vivant, de rendre sensible la vivacité du modèle au moment de la création et sur l'objet achevé. Associé à la sculpture, le portrait sur le vif suppose de faire l'empreinte d'un visage et d'en dégager un moule, qui portera la trace de l'exactitude des traits. Pline l'Ancien raconte que

celui qui, le premier de tous, fit un portrait d'homme avec du plâtre, en prenant un moulage sur le visage même, puis imagina de verser de la cire dans ce moule de plâtre, cire sur laquelle il procédait à des retouches, fut Lysistratus de Sicyone, frère de Lysippe, dont nous avons parlé. Ce fut lui aussi qui instaura la pratique de rendre la ressemblance; avant lui, on s'appliquait à faire les visages aussi beaux que possible ${ }^{11}$.

À l'époque de la Renaissance, quand les critères de beauté sont (paradoxalement) fondés sur la juste imitation des choses, la reconnaissance d'un portrait sur le vif démontre

\footnotetext{
${ }^{11}$ Pline l'Ancien, Histoire Naturelle Livre XXXV, La Peinture, 12 (44), Paris, Les Belles Lettres, 1997, p. 135.
} 
son absolue ressemblance au modèle et apporte la preuve du caractère vivant de l'imitation. C'est pourquoi cette pratique du portrait est un comble du portrait, qui porte la ressemblance au pinacle du genre, duquel il ne se départira pas. Comment reconnaître de telles conditions de réalisation? D’une part, dans le façonnement de l'œuvre, dont rend compte le matériau utilisé, d'autre part, dans les témoignages qui accompagnent sa réalisation, dont celui de Cennino Cennini à la fin du Trecento, fournit un exemple éloquent. Il y est décrit 1) «comment on moule d'après nature le visage d'un homme ou d'une femme $»^{12}$ : « Prends de l'huile parfumée à l'essence de roses ; avec un pinceau de petit-gris, plutôt gros, oins le visage; mets sur sa tête un bonnet ou un capuchon; prends une bande large d'un empan et longue d'une épaule à l'autre; entoures-en le somment de la tête, sur le bonnet, et couds le bord, autour du bonnet, d'une oreille à l'autre $[\ldots]$ » 2) 《comment on permet de respirer à la personne dont on moule la figure $»^{13}:$ « Tu dois faire exécuter par un orfèvre deux tubes de cuivre ou d'argent, qui soient ronds en haut et plus ouverts qu'en bas, comme une trompette [...]»;3) « comment on coule le moule en plâtre sur la personne vivante; comment on l'enlève, comment on le conserve et on le coule en métal ${ }^{14}$ : «Prends un verre et un peu de cette préparation. Quand tu l'as rempli de façon égale, réserve les yeux pour les couvrir, après tout le visage. Fais tenir la bouche et les yeux fermés, sans effort car ce n'est pas nécessaire, [...] Quand ta préparation est bien sèche, avec un canif ou un petit couteau ou des ciseaux, enlève délicatement tout autour la bande que tu as cousue ; retire les petits tubes de son nez, avec précaution $[\ldots] »^{15}$ (fig. 11).

Fig.11.

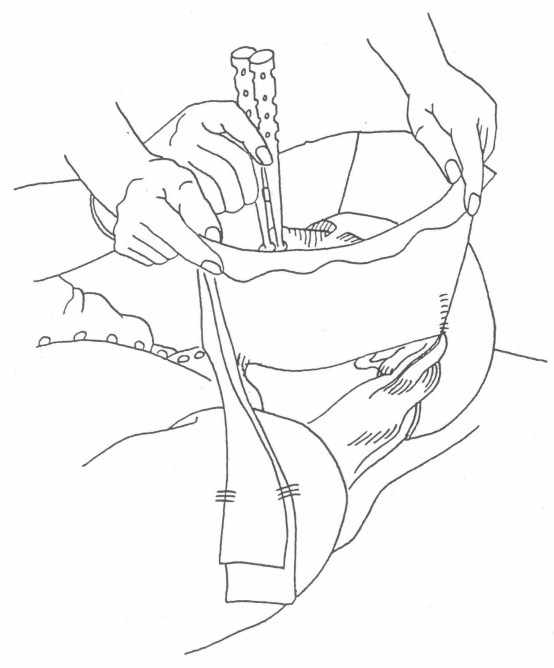

Cennino Cennini, « Comment on permet de respirer à la personne dont on moule la figure » (figure extraite de Le livre de l'Art (Il libro dell'arte), op.cit.)

\footnotetext{
${ }_{12}$ Cennino Cennini, Le Livre de l'Art (Il libro dell'arte), (tr. Colette Deroche), Paris, Éditions Berger-Levrault, 1992, p. 324. L'ouvrage est écrit à la fin du XIVe siècle ou au début du XVe siècle.

${ }^{13}$ Ibid., p. 327.

${ }^{14}$ Ibid., p. 329.

${ }^{15}$ Ibid., p. 330. Pour un commentaire du texte de Cennini, dans son lien avec le portrait sur le vif, voir Georges Didi-Huberman, « Ressemblance mythifiée et ressemblance oubliée chez Vasari : la légende du portrait "sur le vif” », Mélanges de l'École Française de Rome Italie et Méditerranée t. 106, 2, 1994.
} 
Fig.12.

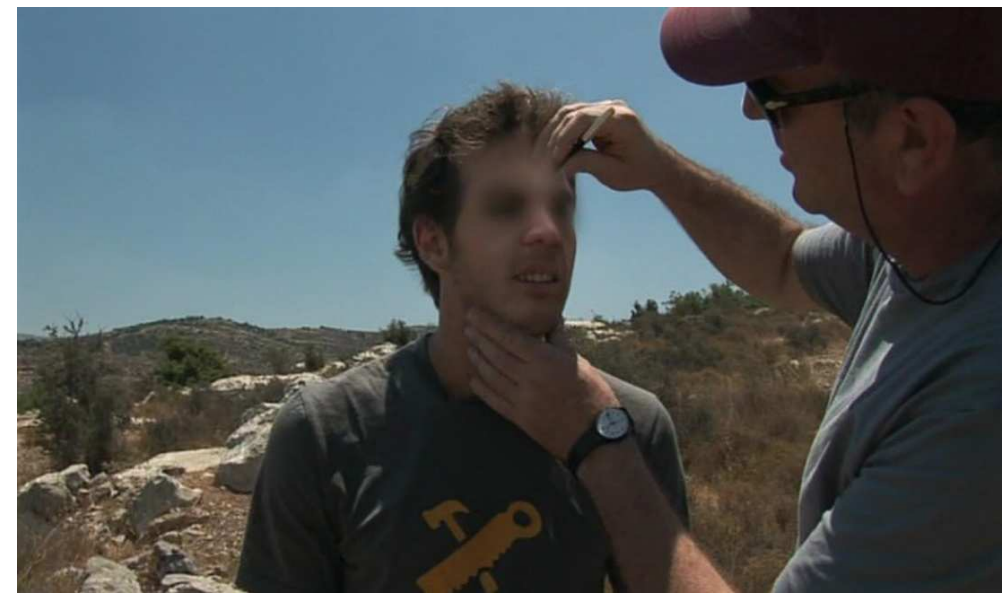

Avi Mograbi, Z32, 2008 - Photogramme du film (dvd)

Tout le film de Mograbi est placé sous le signe de la réalisation du portrait. Il s'ouvre sur la présentation par le cinéaste de sa façon de faire - celle-ci fût-elle décalée par l'ironie de la mise en scène ${ }^{16}$. Si les transformations du visage, jamais commentées, jamais dites d'aucune sorte, rendent visible le travail de façonnage technique, une scène témoigne explicitement de cet art que l'on désigne par le verbe «mouler », rappelle Cennini, cet art « qui est très utile au dessin et qui te fera grand honneur, en te faisant copier et imiter les choses d'après nature ${ }^{17}$. Dans la dernière partie du récit, alors que le cinéaste a emmené le soldat sur les lieux du crime - un terrain vague laissé à l'abandon, écrasé par la lumière du soleil - on voit le moulage en train d'être fait. Tout à coup, Mograbi s'approche de Z32 et avec une sorte de stylo, il fait des points sur son visage - jamais autrement visibles au spectateur que par l'intermédiaire de légères traces lumineuses (fig.12). Au point d'acmé du récit, celui de la reconstitution du geste criminel, et alors même que le masque est au comble de sa ressemblance, le cinéaste figure à même son modèle l'empreinte à l'origine du moule, comme si le geste de création faisait obstacle à l'emportement du jeune soldat dans la redécouverte jouissive des gestes exécutés. Mograbi raconte que «sur place [les lieux du crime], il y avait en lui comme une opacité. Il semblait ne rien voir. Il ne "voit" pas la femme palestinienne qui traverse le champ. En revanche, il bondissait partout comme si son corps retrouvait tous les gestes de ce moment terrible et même le plaisir qu'il en avait retiré $»^{18}$. Rappeler que cette vivacité du soldat est aussi la matière première du moule qui est en train d'être fait, est une manière de rendre visible cet aveuglement, en faisant du geste créatif la seule réplique possible au discours du meurtrier. Il n'est pas jusqu'au texte récent d'Avi Mussel sur l'élaboration des trucages et le travail avec le cinéaste, qui n'évoque la technique du portrait sur le vif dans le nécessaire commentaire du travail, en écho au texte de Cennini - comme s'il fallait que la description scrupuleuse de la confection du moulage apporte une preuve supplémentaire du travail sur empreinte. Il est surprenant de constater combien la présentation détaillée des étapes du travail, des épreuves surmontées et finalement de la prouesse technique accomplie, est proche du texte de la Renaissance jusque dans les plus infimes parties de l'opération ${ }^{19}$. Mais ce qui frappe, surtout, c'est que la technique

\footnotetext{
${ }^{16}$ Notons que le film produit l'impression constante d'un work in progress; d'ailleurs, le générique a lieu au bout d'une demi heure.

${ }_{17}$ Ibid., p. 323-324.

${ }^{18}$ L'Humanité, 18 février 2009.

${ }^{19}$ En effet, dans les deux cas, l'un des problèmes posés à la confection du masque provient de la barbe. Cennini recommande qu'elle soit bien rasée, quand de son côté, A. Mussel explique: «Un petit problème parmi d'autres : le personnage principal n'était pas rasé. À cause des jours passés entre un tournage et le suivant, ses poils de barbe étaient de longueur différente selon les plans et un même masque ne suffisait pas [...]», «Dissimuler pour révéler ou l'effet du documentaire » («Dissimuler », art.cit., p. 62).
} 
sophistiquée, qui consiste à reproduire les déplacements du visage grâce à un logiciel d'animation $3 \mathrm{D}$ et, ensuite, à les pister via un programme d'ordinateur spécialement conçu à cet effet, prend appui sur un geste manuel très simple, qui consiste à dessiner des points au crayon sur le visage du personnage. Si trois spécialistes des effets spéciaux ont travaillé cinq jours par semaine pendant neuf mois, englobant l'essentiel des dépenses allouées pour le film, c'est donc finalement pour reconduire les deux traits fondamentaux du portrait sur le vif - l'origine esthétique de sa fabrique, l'indispensable ressemblance au modèle - à la faveur du récit.

\section{La double scène du sujet}

Motif récurrent du cinéma de Mograbi : la mise en scène du cinéaste par lui-même qui atteint dans $Z 32$ un degré de réflexivité vertigineux ${ }^{20}$. On a dit déjà combien le début du film étonne, qui expose le cinéaste en personnage de lui-même, incarnant le rôle de celui qui ne sait comment rendre possible (filmable) ce témoignage. Avant même que Mograbi s'explique, nous voyons - et ce sont les premières images du film - le soldat et sa compagne se demander si «ça tourne », question reprise scène suivante par Mograbi lui-même, sa cagoule sur la tête. De façon générale, soldat et cinéaste sont représentés de manière comparable, soit que leurs paroles se répètent ou se répondent, soit que le cadrage qui les expose soit le même, de sorte qu'au lieu d'un face à face, d'un jeu de questions-réponses à laquelle la situation prédispose, un aller-retour s'effectue entre les deux hommes. La possibilité du témoignage importe tout autant que le témoignage lui-même, les conditions, morales et éthiques, d'une telle rencontre deviennent elles-mêmes l'enjeu de la mise en scène. Ce qui explique que le cinéaste multiplie les postures énonciatrices. Il chante ses doutes, d'abord avec un pianiste, qu'il fait répéter, ensuite avec un orchestre, qu'il invite chez lui, dans son salon. Toujours en chantant, il met en scène la parole de sa femme : "C'est pas un sujet pour un film [...] Il joue le rôle du repenti, tu fais celui qui observe. Il se lave à ton regard, tu t'en tires encore avec un film percutant ». On remarque que cette manière de faire de la possibilité de l'œuvre le sujet de l'œuvre est une démarche artistique classique qui renoue avec les avant-gardes du $\mathrm{XX}^{\mathrm{e}}$ siècle. Toutefois, les interruptions répétées de l'action principale par l'orchestre du salon, l'alternance entre le récit du soldat et son commentaire chanté par Mograbi produisent un effet de distanciation (comique) qui fait surgir l'image d'une ancienne pratique théâtrale : celle de la tragédie grecque et de sa relecture historique par le théâtre épique de Brecht ${ }^{21}$. À ceci près que la scission du sujet à l'œuvre dans ces deux formes, classique et moderne, de théâtre réflexif, conduit à la mise en évidence de la figure, elle-même divisée du cinéaste, dont l'action politique est convertie ici en un geste de création. Et, de fait, l'échange entre les deux hommes ne s'effectue pas que dans un sens. Si Mograbi n'hésite pas à incarner son propre rôle pour devenir personnage à part entière, il laisse au soldat la possibilité de filmer son propre récit, de sorte que le questionnement éthique qui circule d'un bout à l'autre du film, prend la forme d'une réflexion sur la mise en scène. "Puis-je héberger un assassin dans mon film et si oui quelle forme plastique donner à ce récit? » demande le cinéaste ; «Quel récit donner à cet événement? » semble répondre le

\footnotetext{
${ }^{20}$ La mise en scène portraitique du cinéaste est un motif structurant de son œuvre, installations comprises. On le retrouve notamment, associé à la figure de l'autofiction, dans Comment j'ai appris à surmonter ma peur et appris à aimer Ariel Sharon, 1996, et surtout dans Août (Avant l'explosion), 2001 - comme le remarque Christa Blümlinger, que je remercie ici d'avoir rendu possible l'écriture de ce texte. Voir «Le peuple qui manque. À propos des installations d'Avi Mograbi », Trafic 72, hiver 2009, p. 64-70.

${ }_{21}$ Ce que Mograbi souligne, à la sortie du film : «Après coup, on voit les références à Brecht et au théâtre épique où le chœur exprime ce que le personnage ne peut exprimer, mais ça n'était pas conscient au départ » (Libération, 18 février 2009).
} 
soldat qui multiplie de son côté les dispositifs narratifs. Les scènes entre Z32 et sa compagne ont été tournées en Inde, en l'absence du cinéaste qui a mis une caméra à leur disposition. Totalement livré à lui-même, le couple décide des plans à tourner, de la place de la caméra, de ce qu'ils disent ou pas - «Arrête de filmer » dit la fille à plusieurs reprises. C'est que le soldat dirige sa compagne : «Raconte-moi mon histoire, lui demande-t-il, comme si tu étais une actrice, comme si tu étais Lady Macbeth ». Et elle de s'exécuter maladroitement, imitant le ton grave de sa voix, reprenant les mots vulgaires qu'elle lui a entendus dire et que, amusé, il répète après elle. "Il essaie de lui faire dire ce que le metteur en scène voudrait entendre, explique Mograbi. Elle exprime ce qui pourrait être le point de vue du cinéaste tout en occupant un rôle important que je ne pouvais remplir $»^{22}$. Au problème du cinéaste, qui se demande comment filmer, se superpose celui du soldat, qui cherche comment raconter ce qui s'est passé. Et la parole de circuler d'un personnage à l'autre soumise à un double effet de réverbération et de dispersion : interrompue, reprise, relayée à satiété. Au lieu de faire de Z32 un personnage auquel s'identifier, Mograbi met le soldat dans la position qui est la sienne, il force cette distance qui l'oblige à se détacher de son récit pour s'interroger sur la forme qu'il faut lui donner. En retour, la parole de culpabilité circule d'un homme à l'autre. Le pardon, réclamé par le soldat à sa jeune compagne, qui ne sait comment lui accorder, devient le fond de la chanson, la préoccupation majeure du cinéaste: «Finalement, qui en tire bénéfice? C'est moi qui tire un bénéfice / de le voir se tourmenter / d'avoir éprouver du plaisir / En vérité, ça me ronge / d'en avoir fait une chanson / qu'il ait réduit un homme à une tâche / et que je puisse lui pardonner ».

Éviter l'empathie, écouter pour mieux comprendre, mais comprendre sans excuser : le cinéaste donne la parole au meurtrier, mais il ne laisse pas cette parole retomber dans le vide. Dans la dernière partie du film, Mograbi accuse la division du sujet en dédoublant la figure du soldat quand sur les lieux du crime celui-ci raconte les faits et en surimpressionnant son image à la sienne - de façon que les visages se côtoient avec évitement des regards, comme dans les portraits pluriels (Jean-Luc Nancy: «Il est d'autant plus remarquable que la caractéristique d'un portrait pluriel soit toujours l'évitement mutuel des regards : ils ne se rencontrent pas et ne se cherchent même pas $\left.{ }^{23}\right)$. Le double portrait est la réponse figurative que le cinéaste apporte au problème éthique soulevé par son film. Il marque cette distance à l'image qui sépare le cinéaste de son sujet - que déjà la scénographie souligne (le théâtre de poche, l'allusion tragique) - à partir d'une béance entre les deux regards, qui permet l'échange sans la rencontre et bloque l'identification. Au lieu d'un contrechamp sur l'événement - toujours invisible à la représentation - appelé par le regard dirigé hors champ, ce jeu d'aller-retour entre les deux visages resserre sur la question du genre l'enjeu de la figuration.

\section{Visage(s) de l'histoire}

Depuis la tradition classique, qui fait remonter aux rites funéraires antiques l'origine du portrait, la ressemblance au modèle est liée à la résistance par l'image à la disparition physique du modèle. Garder une image pour l'éternité et ne pas hésiter à revoir certains attributs de la personne afin qu'ils soient plus conformes à l'idée que d'elle on souhaite pérenniser. Dans Z32, la résistance à la disparition physique est dite par le dialogue («Tu as épongé ta sueur? » demande Mograbi au soldat en s'approchant de lui pour rectifier avec son stylo les marques du visage nécessaires à la fabrication numérique du masque - «Non,

\footnotetext{
${ }^{22}$ L'Humanité, art.cit..

${ }^{23}$ J.-L. Nancy, Le Regard du Portrait, op.cit., p. 20.
} 
mon corps résiste - répond le soldat, il ne se laisse pas effacer »), mais elle est dotée d'une acception nouvelle, qui associe le problème de visibilité à l'acte de résistance politique, qui encourage le soldat à témoigner en dépit de ses craintes. Si du portrait revient le dilemme de la ressemblance, tendu entre ce qui reste caché et ce qui tend à apparaître, entièrement voué au combat moral que se livrent ensemble le soldat et le cinéaste, la dissemblance devient figure de résistance - cette lutte du sujet, l'expression éthique d'une reconquête existentielle, bien au-delà de la question du pardon. Comme toujours dans le portrait, il importe de faire coïncider la subjectivation et la figuration - à ceci près que dans le film celle-ci est tenue à la définition politique d'un trait d'humanité de l'homme - son destin anthropologique.

Ce portrait est bien celui d'une perte et, à ce titre, il renvoie à de nombreuses pratiques de dévisagement, auxquelles le Xxe siècle nous a habitués. Le flou qui camoufle tout le visage évoque le reportage journalistique, mais rapidement il dérive, écarté par le cinéaste, comme si les images trop vues de la télévision tiraient le visage vers autre chose. La « défaite du visage $»^{24}$ dans l'histoire de sa représentation a rencontré de nombreuses formes, liées à la perte de noblesse assignée au portrait humaniste. Avec sa ressemblance, le portrait perd la maîtrise de son regard et l'assurance en son image. Dès la fin du XIX siècle, photographie judiciaire et médicale opèrent, par une mise en série comparatiste des visages, une prise de pouvoir sur la personne qui annule la singularité du sujet. Par la suite, l'atteinte portée au visage reste toujours dépendante d'une mutation du sujet, en peinture tout d'abord, mais aussi en photographie et au cinéma, soit que cette atteinte s'associe à un égarement du sens qui n'est pas étranger aux fondements anthropologiques des sociétés capitalistes, soit qu'elle s'inscrive dans une culture post-traumatique à laquelle le film renvoie aussi. Au-delà des formes plurielles que le portrait donne à cette défaite, il importe, dès la deuxième partie du $\mathrm{XX}^{\mathrm{e}}$ siècle, de faire porter au visage la responsabilité de son histoire. Pour le dire sans doute un peu vite : le visage défait dit la défection du monde et si la représentation, moderne, du visage ne se réduit certes pas à ce symptôme-là, il n'empêche que c'est lui qui se voit le plus : paradoxalement, l'expression du vide s'affiche. Depuis les années 1980, le portrait photographique multiplie les tentatives de dépersonnalisation, qu'il recoure à l'usage du flou (Florence Chevalier, Troublé en Vérité, 1981 - fig.13), à l'hypertrophie d'un organe comme la bouche (Ann Mandelbaum, Sans titre, 1992 - fig.14), au mixage d'images sur ordinateur (Nancy Burson, Sans titre, 1988 - fig.15), ou à la rature du visage (Vincent Cordebard, Sans titre, extrait de la série «Conversations faites à un enfant mort », 1991-1992 - fig.16) autant de pratiques introduites par le cinéma, où le visage est devenu le lieu possible de toutes les altérations, dès lors que le montage a donné les bases expressives du morcellement du corps.

\footnotetext{
${ }^{24}$ Jacques Aumont, Du Visage au Cinéma, Paris, Cahiers du Cinéma, coll. « essais », 1992 (« Le visage défait », p. 149-169) et André Rouillé, «Éclipses du visages », La Recherche Photographique 14, " Dévisager », printemps 1993, p. 6. Sur la dé-personalisation du portrait dans la photographie contemporaine et ses liens avec le visage, voir l'article de William Ewing, « Making Faces the Portrait is Dead. Long live the Face! », Next Level 4, 2004 (ou en ligne sur le site du musée de l’Élysée à Lausanne : http://www.elysee.ch).
} 
Fig.13.

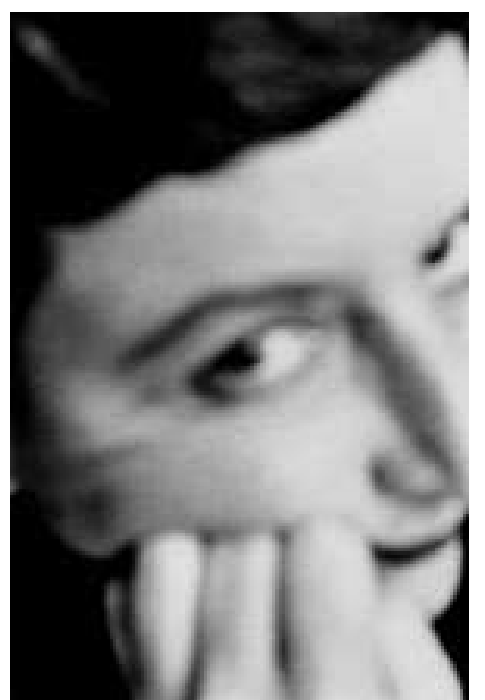

Florence Chevalier, Troublé en Vérité, 1981

Fig.14.

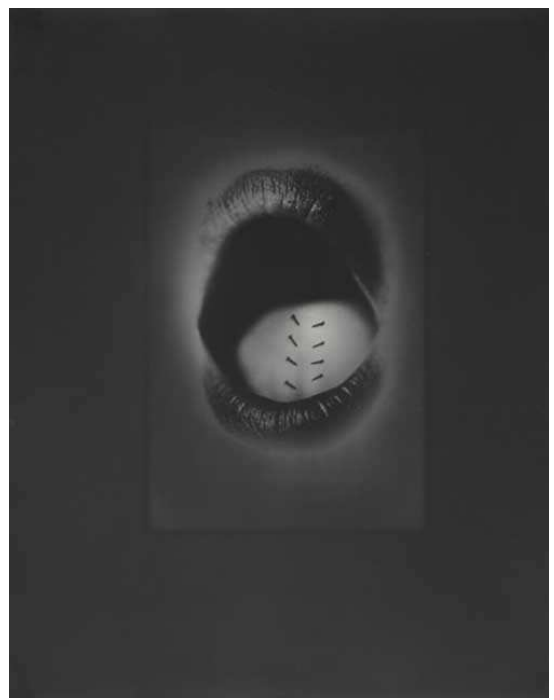

Ann Mandelbaum, Sans titre, 1992

Fig.15.

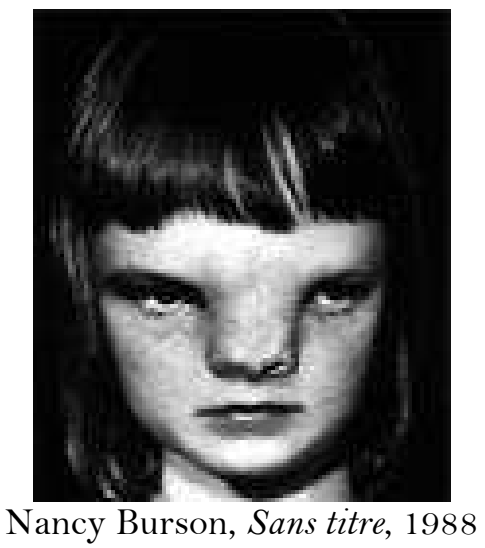


Fig.16.

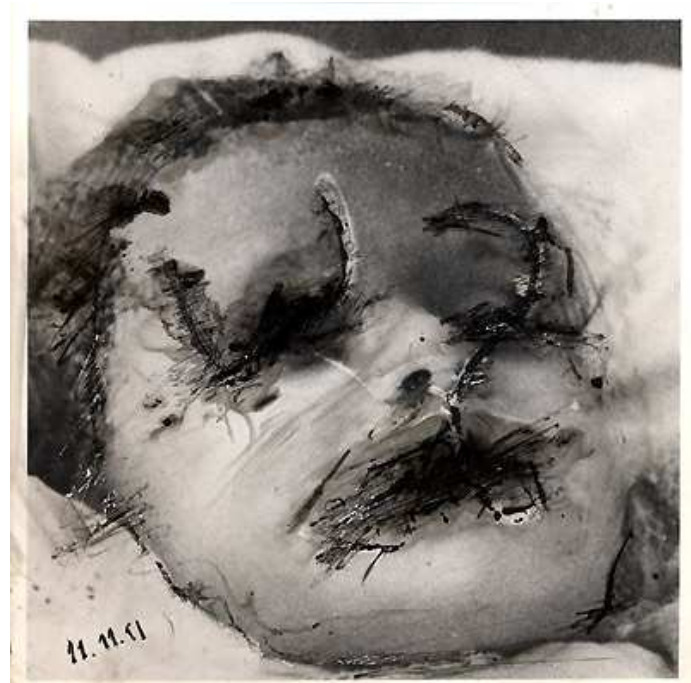

Vincent Cordebard, Sans titre, extrait de la série « Conversations faites à un enfant mort », 1991-1992

D'où vient alors cette impression que ce portrait-là est totalement neuf, en dépit des affinités que les trois masques de Z32 entretiennent avec ces pratiques (télévisuelles, photographiques, cinématographiques) aujourd'hui courantes? Au déplacement visuel de la parole, à la façon dont ce qui est dit remonte à la surface sensible du visage, faisant surgir un fond d'images défuntes. C'est cette migration du discours sur le terrain de la sensation c'est-à-dire de l'art (du portrait) - qui témoigne de la transformation des visages, par-delà deux usages du portrait de cire, associées à deux pratiques que l'histoire a fait se rencontrer : le masque mortuaire et le portrait votif.

Envisagé comme origine du portrait, le masque mortuaire est associé dans l'Antiquité à des rituels funéraires qui célèbrent la trace à jamais ressuscitée du visage de la personne décédée. De là à considérer que tout portrait est lié à une forme de pétrification mortuaire, il n'y a qu'un pas, franchi par Julius von Schlosser qui insiste sur la façon dont les portraits de cire saisissent l'instant de la pétrification, l'étincelle de vie au moment de la mort, qui atteste de la ressemblance à l'image (fig.17). Que l'évocation d'un masque mortuaire soit reconnaissable sous les traits du soldat - dans la conjonction d'une rigidité des traits $\left(2^{\text {e }}\right.$ masque), de leur lissage ( $3^{\text {e }}$ masque) et de la saillie d'une expressivité, par les yeux et par la bouche - semble probable. Ce qui accuse toutefois l'effet de rencontre provient de ce que l'alliance faciale de la vie et de la mort est prise en charge par le dialogue. La mort est le point d'arrivée du récit - le meurtre du policier palestinien - mais aussi son origine - sa manière d'être est ce qui a motivé le film. Elle est donnée dès le début comme la finalité de l'entraînement militaire, la justification de la présence des soldats. Or, le discours du jeune homme associe toujours la perte de sens à laquelle conduit la répétition des gestes de la guerre quand ils deviennent des gestes quotidien, et l'enthousiasme, l'excitation, l'envie et le désir, en bref, l'élan de la vie, comme si au cours de cette période d'enfermement et d'exercices la vie n'avait plus d'autre finalité que cette mort qui lui tend les bras. 
Fig.17.

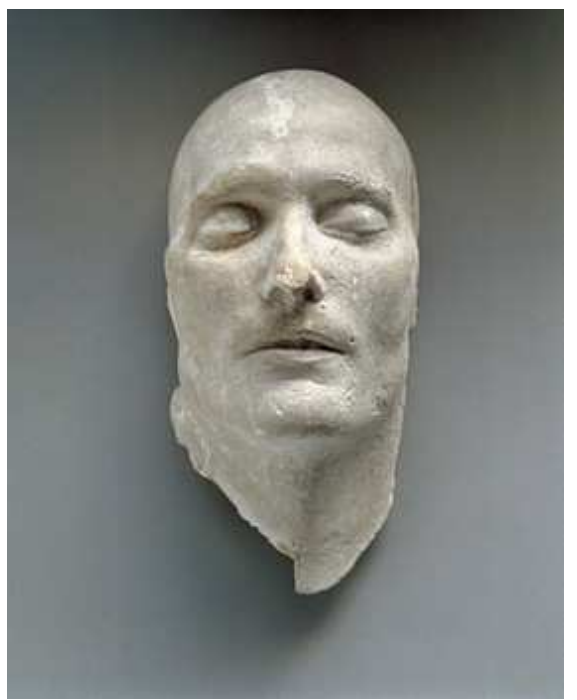

Masque mortuaire de Napoléon, 1821 (origine controversée)

Le portrait de cire a connu un autre usage dans l'Antiquité d'abord puis au Moyen Âge: il fait partie des offrandes votives reproduisant la personne entière ou une partie seulement du corps, qu'un donateur apporte à l'église à la suite d'un vœu en remerciement d'une grâce, pour la guérison d'une infirmité par exemple. Comme la sculpture funéraire, le portrait votif est lié à son modèle par un contrat de ressemblance, au plus près de la nature et de la vie qui palpite. Le phénomène est très populaire dans l'Italie médiévale, où de grands sanctuaires religieux accueillent ces boti, comme on les appelle dans le dialecte florentin, posés contre les murs ou suspendus au plafond. Ils se distinguent des autres portraits par la technique utilisée pour leur fabrique : dal vivo, sur le vif, moulés directement sur le corps du donateur. Leur popularité est considérable à Florence comme en témoigne le cas bien connu de l'église miraculeuse de l'Annunziata, où des centaines de cires sont entreposées, accrochées par des cordes, au point que plusieurs accidents ont été signalés, entrainant au XVI ${ }^{e}$ siècle l'évacuation de l'église jugée trop dangereuse, puis sa restauration en 1664 . L'usage de ces ex-voto est alors si populaire que les marchands des rues adjacentes fabriquent des boti di cartoni (boti en papier mâché) et les vendent pour une somme modique : le plus souvent un membre du corps incarne le vœu et lui donne la forme du lieu où s'exercer, en échange du soulagement souhaitée ${ }^{25}$. Parmi les portraits de cire, la tendance fondamentale est partout la même, note von Schlosser : un «naturalisme atroce $»^{26}$ qui pousse la fidélité au vivant jusqu'à l'indiscrétion car un soin particulier est accordé à la restitution de l'épiderme, les poils et la barbe très scrupuleusement reproduits. C'est aussi ce qui étonne chez Z32 : la quasi perfection de sa ressemblance, qui dans la dernière partie du récit incite le spectateur à scruter les traits de défaillance - soigneusement restitués par le cinéaste ${ }^{27}$ - et à les identifier comme des signes de façonnage. Est troublante cette restitution du vrai (de l'expression) par le faux (du matériau), et plus encore sa dépendance à la parole votive du soldat. En effet, le soldat émet un vœu que tout son discours contient et qui explique qu'il se rende disponible

\footnotetext{
${ }_{25}$ Voir Georges Didi-Huberman, Ex-voto Image, Organe, Temps, Paris, Bayard, Hors collection, Le rayon des curiosités, 2006.

${ }_{26}$ J. von Schlosser, Histoire du Portrait en Cire, op.cit., p. 97.

27 «C'est que ce dernier masque est seulement presque parfait. La technologie dont nous disposons nous aurait permis d'obtenir un résultat indiscernable de la vraie peau. Mes assistants de laboratoire avaient spontanément commencé à travailler dans cette direction. Je les ai arrêtés, car de petites imperfections étaient indispensables pour garder la trace de l'illusion. [...] Il était techniquement possible de faire passer leurs mains sur le masque. Je me suis arrangé pour que leurs mains, passant au-dessous du trucage, en révèlent la présence. Le premier travail consiste à créer une illusion, le $2^{\mathrm{e}}$ à la casser » (A. Mograbi, Cahiers du Cinéma 639, art.cit.).
} 
comme modèle : qu'on lui pardonne. Il le dit et le répète à la caméra, à sa compagne ${ }^{28}-$ et sa requête résonne, qui trouve un écho dans la bouche du cinéaste (peut-il lui pardonner / se pardonner d'abriter un assassin dans son film). En d'autres termes, ce qui revient n'est pas tant une parenté figurale que la façon dont celle-ci est articulée, par la parole, à l'histoire qui l'a vue naître. La tension saisissante entre la mortification des traits que le masque suggère et le souffle de la vie transmis par les orifices du visage est tout entière tendue entre la formulation du vœu et la quête de ressemblance à laquelle le film conduit. Et peu importe que le visage final ne soit pas celui du soldat puisque le pacte de non reconnaissance est respecté - dans le portrait « la ressemblance n'a rien à voir avec la reconnaissance » ${ }^{29}-$ le visage ne cesse de ressembler en vertu des termes du contrat votif qui unit le donateur (Z32) au lieu de l'offrande (le film), offert, comme autrefois, au désir d'être entendu (par Dieu, par le spectateur), grâce à la mise en spectacle de sa personne. Ainsi, masque mortuaire et portrait votif sont-ils les manifestations visibles de la résistance politique à l'effacement du soldat, que la parole produit, distincte, précise. Ici, les mots reconfigurent numériquement le visage par la mise en place d'une dynamique de déplacement des formes, au cour de la ressemblance.

Pour penser l'actualité de l'histoire, l'histoire en train de se faire, dans l'urgence de ses interrogations politiques, le film rend opératoires d'anciens problèmes de figuration. L'invention numérique active une critique efficace de la figure, en lien direct avec l'économie du visage au cœur de l'histoire des civilisations. Résultat de la conception graphique : des images défuntes refont surface, qui relancent les problèmes anthropologiques de leurs origines et les déplacent sur le terrain de l'actualité conflictuelle : elles répondent à l'urgence - historique - de redéfinir une poétique de l'homme qui soit aussi une politique du sujet.

Emmanuelle André est maître de conférences en études cinématographiques à l'université Paris-Diderot, auteur de Esthétique du Motif. Cinéma, musique, peinture, Presses Universitaires de Vincennes, 2007 et d'un ouvrage à paraître intitulé Le Choc du Sujet. De l'hystérie au cinéma (XIX ${ }^{c}-X X I^{e}$ siècles). Ses travaux récents portent sur les liens entre l'esthétique du cinéma, l'histoire de l'art et l'anthropologie culturelle.

\footnotetext{
${ }_{28}$ Auprès d'elle, Z32 supplie presque et l'on comprend bien que pour le couple, l'enjeu est crucial : «J'ai l'impression d'implorer ton pardon. J'avais envie que tu me pardonnes. Je te demande de me pardonner. » Elle lui répond : «Aujourd'hui je suis prête à te pardonner... C'est nouveau chez toi. Au début, tu voulais seulement être compris $[\ldots]$ Mon pardon, c'est de te considérer comme un adulte qui prend de la distance, qui comprend, est en colère avec tout ça ».

29 J.-L. Nancy, Le Regard du Portrait, op.cit., p. 40.
} 